\title{
A DIVERSITY ACCOUNT OF BRYACEAE (BRYOPHYTA: MUSCI) OF NEPAL
}

\author{
N. Pradhan and S. D Joshi
}

\begin{abstract}
Bryums are diverse mosses distributed widely throughout the country. The species diversity of Bryaceae which were documented in various periods has been presented here. This paper includes 82 species including three subspecies and five varieties under seven genera of the total recorded 1150 bryofloral species in Nepal so far. Besides published records, the field survey of the first author within last five years has also been included here.
\end{abstract}

Key words: Bryoflora, cosmopolitan, diversity, distribution, species

\section{Introduction}

Bryaceae is a large and cosmopolitan family which includes genera of considerable taxonomic difficulties. Basically, this family has interesting genera with odd gametophytic structures including acrocarpous stems and unicostate leaves with acute apex. This is a small to robust plant, commonly known as Hump moss, Silver moss, Thread moss, etc. It bears an erect solitary or branched stem. Leaves are arranged spirally and are usually small and distant below, equally spaced and occasionally form a rosette or structured differently. Gemmae are often present. Plant may be autoecious or dioecious, sometimes synoecious. Sporophytes generally bear pendulous capsules which are spherical and lightly papillose. Andrew (1935) suggested that the family might best be understood as a single large natural genus Bryum.

Mitten (1859) has enumerated 52 species of Bryum including 17 species from Nepal. Noguchi et al. (1966) enumerated 13 species of Bryum which were collected by Dr. Yoda during the East Himalayan Expedition in 1963. The second work of Noguchi (1966) during the first phase of the East Himalaya Expedition in 1960 and 1963 came up with a list of 26 species and four varieties including 14 species from Nepal. Noguchi's next work (1971) added three more species collected during the second phase Expedition of the Eastern Himalaya. Gangulee (1974) recorded 35 species in eastern Nepal.

The notable work of Iwatsuki (1979a) brought out a list of 13 species of bryaceae from Central Nepal which was reported during Kochi Himalaya Expedition of 1976. Similarly, the East Himalayan Expedition of Chiba University in 1977 added 18 more species (Iwatsuki $1979 b)$ to the overall list. Hedderson and Harold (1990) has added a new moss species Plagiobryum duthiei Broth. ex. Hedderson \& Harold recorded from western and central Nepal.

Other notable works on Nepalese Bryaceae were done by Karczmarz (1981) who reported five species in Kathmandu.Valley. Higuchi and Takaki (1990) enumerated 41 species in the central and eastern regions. Kattel and Adhikari (1992) presented a list of 62 species and two varities in their publication. The recent work of Pradhan (2000a) brought out a list of 78 
species and four varities under six genera collected at different localities of the country.

Pradhan (2000b) in her study recorded 15 species of Bryum from Phulchowki of Central midland. Likewise, Pradhan and Shrestha (2002) recorded 26 species from the alpine zone. Long (1995) made an interesting study on the type localities of some bryaceae occurring at different ecological zones of the country.

\section{Materials and Methods}

Various habitat preferences can be found among the members of this family. Specimens were collected by peeling off the substrate; generally a small knife was used very carefully for this purpose. All the collected materials were placed in hand made paper packets with proper field notes. These specimens were dried in mild sun for an hour or so to evaporate moisture content of the specimens. These specimens later were identified at the Natural History Museum, Swayanbhu and Central Department of Botany, Tribhuvan University, Kathmandu. Some specimens were also tallied in Museum for these specimens besides consulting relevant literatures like Watson and Richards (1968), Gangulee (1974), Nyholm (1974), Chopra (1975), Eddy (1996), and Allen (2002). The author's names were checked consulting Brummitt and Powell (1992). The website of MOBOT was used to check the accepted names.

\section{Results}

The report includes 82 species including three subspecies and five varieties belonging to seven genera of Bryaceae which are provided below in tabulated form. This also includes some species recorded by the foreign expedition team to the central and east Himalayas, besides authors' own field research across the country. The distribution of every species has been provided region wise with altitudinal ranges and status category. They are arranged alphabetically.

Table1. Species diversity of Bryaceae in listed form

\begin{tabular}{|c|c|c|c|c|c|c|c|}
\hline \multirow[t]{2}{*}{$\begin{array}{l}\text { S. } \\
\text { No. }\end{array}$} & \multirow[t]{2}{*}{ Scientific names } & \multicolumn{3}{|c|}{$\begin{array}{c}\text { Distributional } \\
\text { Ranges }\end{array}$} & \multirow{2}{*}{$\begin{array}{c}\text { Altitudinal } \\
\text { Differences } \\
\text { m. }\end{array}$} & \multirow[t]{2}{*}{ Status } & \multirow[t]{2}{*}{ Remarks } \\
\hline & & $\mathbf{W}$ & C & $E$ & & & \\
\hline 1 & $\begin{array}{l}\text { Anomobryum auratum (Mitt.) A. Jaeger } \\
\text { Bryum auratum Mitt. }\end{array}$ & & + & + & $1700-2900$ & $\mathrm{R}$ & \\
\hline 2 & $\begin{array}{l}\text { Anomobryum cymbifolium (Lindb.) Broth. } \\
\text { Brachymnium filiforme Griff. }\end{array}$ & + & & & & $T$ & \\
\hline 3 & $\begin{array}{l}\text { Anomobryum filiforme (Dicks.) Husn. } \\
\text { Bryum filiforme Dicks. }\end{array}$ & & + & + & $2000-2200$ & $\mathrm{R}$ & \\
\hline 4 & $\begin{array}{l}\text { Anomobryum filiforme ssp. concinnatum } \\
\text { (Spruce) Loeske } \\
\text { Bryum filiforme var. concinnatum (Spruce) } \\
\text { Boulter }\end{array}$ & & + & + & $1500-41000$ & C & \\
\hline 5 & $\begin{array}{l}\text { Anomobryum germigenum Broth. } \\
\text { Bryum germigerum (Broth.) E. B. Bartram }\end{array}$ & & + & & $1200-2700$ & $\mathrm{M}$ & \\
\hline 6 & $\begin{array}{l}\text { Anomobryum polymorphum Dixon } \\
\text { Bryum indicopolymorphum Ochi }\end{array}$ & & + & + & $1600-2100$ & $\mathrm{R}$ & \\
\hline 7 & $\begin{array}{l}\text { Anomobryum yasudae Broth. } \\
\text { Bryum yasudae (Broth.) Ochi }\end{array}$ & & & + & 1350 & $\mathrm{R}$ & \\
\hline 8 & Brachymenium acuminatum Harv. & & + & + & $250-3000$ & $T$ & \\
\hline 9 & Brachymenium bryoides Hook. & & & + & $1900-2000$ & $T$ & \\
\hline 10 & Brachymenium capitulatum (Mitt.) Kindb. & & + & + & $1500-2600$ & C & \\
\hline 11 & $\begin{array}{l}\text { Brachymenium indicum (Dozy \& Molk.) } \\
\text { Bosch \& Lacey }\end{array}$ & & & + & 2720 & $\mathrm{R}$ & \\
\hline
\end{tabular}


Pradhan and Joshi : A Diversity Account of.

\begin{tabular}{|c|c|c|c|c|c|c|c|}
\hline \multirow[t]{2}{*}{$\begin{array}{l}\text { S. } \\
\text { No. }\end{array}$} & \multirow[t]{2}{*}{ Scientific names } & \multicolumn{3}{|c|}{$\begin{array}{c}\text { Distributional } \\
\text { Ranges }\end{array}$} & \multirow{2}{*}{$\begin{array}{l}\text { Altitudinal } \\
\text { Differences } \\
\text { m. }\end{array}$} & \multirow[t]{2}{*}{ Status } & \multirow[t]{2}{*}{ Remarks } \\
\hline & & $\mathbf{W}$ & C & $\mathrm{E}$ & & & \\
\hline 12 & Brachymenium longicolle Ther. & & + & + & $1700-3400$ & $\mathrm{R}$ & \\
\hline 13 & $\begin{array}{l}\text { Brachymenium longidens Renner \& M. A. } \\
\text { Cardenas }\end{array}$ & & + & & 3700 & $\mathrm{R}$ & \\
\hline 14 & Brachymenium microstomum Harv. & & & & & $T$ & \\
\hline 15 & Brachymenium nepalensis Hook. & & + & + & $1500-2900$ & $\mathrm{M}$ & \\
\hline 16 & Brachymenium ochianum Gangulee & & & + & $2000-2600$ & $\mathrm{M}$ & \\
\hline 17 & Brachymenium ptychothecium (Besch.) Ochi & & + & & $3600-4200$ & $\mathrm{R}$ & \\
\hline 18 & Brachymenium splachnoides Harv. & & & & & $T$ & \\
\hline 19 & Bryum algovicum Sendtn. ex C. Muell. & & & + & 3900 & $\mathrm{R}$ & Erosion control \\
\hline 20 & Bryum alpinum Huds. ex With. & & + & & 3300 & $\mathrm{C}$ & \\
\hline 21 & Bryum angustirete Kindb. & & + & & 4400 & $\mathrm{R}$ & \\
\hline 22 & $\begin{array}{l}\text { Bryum apiculatum Schwaegr. } \\
\text { B. plumosum Dozy \& Molk. } \\
\text { B. nitens Hook. }\end{array}$ & & + & + & $150-4400$ & $\mathrm{R}$ & \\
\hline 23 & $\begin{array}{l}\text { Bryum argenteum Hedw. } \\
\text { Bryum argenteum var. lanatum (P. Beauv.) } \\
\text { Hampe }\end{array}$ & + & + & + & $800-5100$ & C & $\begin{array}{l}\text { Food values } \\
\text { (birds); heavy } \\
\text { metal detector; } \\
\text { Ornamental } \\
\text { value (bonsai) }\end{array}$ \\
\hline 24 & $\begin{array}{l}\text { Bryum atrovirens Vilh. ex Brid. } \\
\text { B. erythrocarpum Schwaegr. }\end{array}$ & & & + & $1600-1900$ & $\mathrm{M}$ & \\
\hline 25 & Bryum billardieri Schwaegr. & & & + & 2600 & $\mathrm{C}$ & \\
\hline 26 & $\begin{array}{l}\text { Bryum blandum Hook. f. \& Wilson spp. } \\
\text { handelii (Broth.) Ochi } \\
\text { B. setschwanicum Broth. }\end{array}$ & & + & + & $3300-4200$ & M & \\
\hline 27 & Bryum bohnhofii C. Muell. ex Broth. & & + & & 3900 & $\mathrm{R}$ & \\
\hline 28 & Bryum caespiticium Hedw. & & + & + & $300-4800$ & C & \\
\hline 29 & Bryum capillare Hedw. & & + & & $900-1300$ & C & $\begin{array}{l}\text { Resistant to } \\
\text { Pollution }\end{array}$ \\
\hline 30 & $\begin{array}{l}\text { Bryum cellulare Hook. } \\
\text { Brachymenium splachnoides Harv. } \\
\text { B. splachnoides (Harv.) Muell. Hal. }\end{array}$ & & + & & $900-1300$ & T & $\begin{array}{l}\text { Common in } \\
\text { lowland }\end{array}$ \\
\hline 31 & Bryum clavatum (Schimp.) C. Muell. & & & + & $350-4850$ & $\mathrm{R}$ & \\
\hline 32 & $\begin{array}{l}\text { Bryum coronatum Schwaegr. } \\
\text { B. doliolum Duby }\end{array}$ & + & + & + & $150-1000$ & C & \\
\hline 33 & $\begin{array}{l}\text { Bryum dichotomum Hedw. } \\
\text { B. bicolor Dixon }\end{array}$ & + & + & + & $500-4600$ & $\bar{M}$ & \\
\hline 34 & Bryum evanidinerve Broth. & & + & & 2000 & $\mathrm{R}$ & \\
\hline 35 & Bryum funariodes Ochi & & + & & $2000-4000$ & M & \\
\hline 36 & Bryum haematoneurum C. Muell. & & + & & $1400-2000$ & $\mathrm{R}$ & \\
\hline 37 & Bryum knowltonii Barnes & & + & & & $\mathrm{R}$ & \\
\hline 38 & Bryum leptotorquescens C. Muell. ex. Broth. & & + & & $3000-3900$ & $\mathrm{R}$ & \\
\hline 39 & Bryum leucophylloides Broth. & & + & & $3000-4000$ & & \\
\hline 40 & Bryum pachytheca C. Muell. & & + & & $1400-1500$ & & \\
\hline 41 & $\begin{array}{l}\text { Bryum pallescens Schleich. ex Schwaegr. } \\
\text { B. tibetanum Mitt. }\end{array}$ & & & + & 5000 & $\mathrm{R}$ & \\
\hline 42 & $\begin{array}{l}\text { Bryum paradoxum Schwaegr. } \\
\text { B. teretiusculum Hook. }\end{array}$ & & + & + & $1600-2900$ & $T$ & \\
\hline 43 & Bryum pellucidum (Dixon \& Badhw.) Ochi & & & + & & & \\
\hline 44 & $\begin{array}{l}\text { Bryum petelotii Thér. \& R. Henry } \\
\text { Brachymenium exile (Dozy \& Molk.) Bosch } \\
\text { \& Lacey }\end{array}$ & & + & + & $1400-2900$ & C & $\begin{array}{l}\text { Soil Ph indicator, } \\
\text { use for bonasai }\end{array}$ \\
\hline 45 & $\begin{array}{l}\text { Bryum pseudotriquetrum (Hedw.) P. Gaertn. } \\
\text { B. ventricosum Relhan }\end{array}$ & + & + & + & $2900-6100$ & $\mathrm{C}$ & Rock builder \\
\hline 46 & $\begin{array}{l}\text { Bryum recurvulum var. flexicaule (C. Muell.) } \\
\text { Ochi } \\
\text { B. flexicaule C. Muell. }\end{array}$ & & & + & $1700-1900$ & & \\
\hline 47 & Bryum ramosum (Harv.) Hook. & & & & & 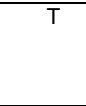 & $\begin{array}{l}\text { Harvey } 124 \\
\text { (TCD) } \\
\text { Wallich H3650 } \\
(\mathrm{BM})\end{array}$ \\
\hline 48 & Bryum reflexifolium (Ochi) Ochi & & & + & $2000-2500$ & & \\
\hline 49 & Bryum retusifolium M.A.Cardenas et P. Vard. & & & + & $1500-1900$ & & \\
\hline
\end{tabular}




\begin{tabular}{|c|c|c|c|c|c|c|c|}
\hline \multirow[t]{2}{*}{$\begin{array}{l}\text { S. } \\
\text { No. }\end{array}$} & \multirow[t]{2}{*}{ Scientific names } & \multicolumn{3}{|c|}{$\begin{array}{c}\text { Distributional } \\
\text { Ranges }\end{array}$} & \multirow{2}{*}{$\begin{array}{l}\text { Altitudinal } \\
\text { Differences } \\
\text { m. }\end{array}$} & \multirow[t]{2}{*}{ Status } & \multirow[t]{2}{*}{ Remarks } \\
\hline & & $\mathbf{W}$ & C & $\mathrm{E}$ & & & \\
\hline 50 & Bryum rostratum Schrad. ex. Sm. & & & + & & & \\
\hline 51 & Bryum salakense M.A. Cardenas & & & + & 2000 & $\mathrm{R}$ & \\
\hline 52 & Bryum subrotundum Brid. & & & + & & & \\
\hline 53 & $\begin{array}{l}\text { Bryum teretiusculum var. reflexifolium (Ochi) } \\
\text { Ochi }\end{array}$ & & & + & $2600-3500$ & C & \\
\hline 54 & Bryum thomsonii Mitt. & & + & + & $2600-4300$ & & \\
\hline 55 & Bryum trachyrhizon C. Muell. & & & + & 4100 & $\mathrm{R}$ & \\
\hline 56 & Bryum turbinatum (Hedw.) Turner & + & & + & $4200-4500$ & $\mathrm{R}$ & \\
\hline 57 & Bryum wrightii Mitt. & & & + & $1800-3800$ & $\mathrm{M}$ & \\
\hline 58 & Mielichhoferia macrophylla Ochi & & + & & 2400 & $\mathrm{R}$ & \\
\hline 59 & $\begin{array}{l}\text { Mielichhoferia mielichhoferi (Hook.) Wijk \& } \\
\text { Margad var. noguchinnum Gangulee }\end{array}$ & & & & & $\mathrm{T}$ & $\begin{array}{l}\text { Zimmermann } \\
\text { 555a (BM) }\end{array}$ \\
\hline 60 & Mielichhoferia sasaokae Broth. & & + & + & $370-4400$ & $\mathrm{R}$ & \\
\hline 61 & Plagiobryum demissum (Hook.) Lindb. & & + & & 3800 & $\mathrm{M}$ & \\
\hline 62 & $\begin{array}{l}\text { Plagiobryum duthiei Broth. ex Hedd. \& } \\
\text { Harold }\end{array}$ & + & + & & $3300-4500$ & $\mathrm{~T}$ & $\begin{array}{l}\text { Voucher } \\
\text { No.634b }\end{array}$ \\
\hline 63 & $\begin{array}{l}\text { Plagiobryum giraldii (C. Muell.) Par. } \\
\text { Bryum giraldii C. Muell. }\end{array}$ & & & + & 3950 & $\mathrm{R}$ & \\
\hline 64 & Pohlia acuminata Hoppe \& Hornsch. & & + & + & $2700-4100$ & C & \\
\hline 65 & $\begin{array}{l}\text { Pohlia camptotrachela (Renner \& } \\
\text { M.A.Cardenas) Broth. }\end{array}$ & & & + & $1100-2100$ & $\mathrm{R}$ & \\
\hline 66 & $\begin{array}{l}\text { Pohlia cruda (Hedw.) Lindb. } \\
\text { Bryum longescen C. Muell. } \\
\text { Webera cruda (Hedw.) Fuener. }\end{array}$ & + & + & + & $3800-4500$ & C & \\
\hline 67 & Pohlia crudoides (Sull. \& Lesq.) Broth. & & + & + & $3200-3900$ & C & \\
\hline 68 & $\begin{array}{l}\text { Pohlia crudoides var. revolvens } \\
\text { (M.A.Cardenas ) Ochi }\end{array}$ & & & + & 4200 & & \\
\hline 69 & $\begin{array}{l}\text { Pohlia elongata Hedw. } \\
\text { Webera elongata (Hedw.) Schwaegr. }\end{array}$ & + & + & & $1500-4400$ & C & \\
\hline 70 & $\begin{array}{l}\text { Pohlia flexuosa Hook. } \\
\text { P. scabridens (Mitt.) Broth. }\end{array}$ & & + & + & $1300-2000$ & $\mathrm{C}$ & \\
\hline 71 & $\begin{array}{l}\text { Pohlia flexuosa var. propagulifera (Renner \& } \\
\text { M.A. Cardenas ) Gangulee }\end{array}$ & & + & & 1500 & C & \\
\hline 72 & $\begin{array}{l}\text { Pohlia leucostoma (Bosch. \& Lacey) M. } \\
\text { Fleisch. }\end{array}$ & & + & + & $1400-2300$ & C & \\
\hline 73 & Pohlia leucostomoides (Broth.) Ochi & & & + & $550-3400$ & C & \\
\hline 74 & Pohlia longicollis (Hedw.) Lindb. & & + & + & $3300-3500$ & C & \\
\hline 75 & Pohlia microstoma (Harv.) Ochi & & & + & $2800-6250$ & $\mathrm{R}$ & $\begin{array}{l}\text { Highest known } \\
\text { alt. record of } \\
\text { Bryaceae in the } \\
\text { world. }\end{array}$ \\
\hline 76 & $\begin{array}{l}\text { Pohlia minor Schieich. ex Schwaegr. ssp. } \\
\text { acuminata (Hoppe \& Hornsch.) Wijk \& } \\
\text { Margad. } \\
\text { Webera acuminata (O. Hopp. \& Hornsch.) } \\
\text { Schimp. }\end{array}$ & & & + & $3000-4300$ & $\mathrm{R}$ & \\
\hline 77 & $\begin{array}{l}\text { Pohlia nutans (Hedw.) Lindb. } \\
\quad \text { Webera nutans }\end{array}$ & & & + & $3600-4200$ & C & \\
\hline 78 & $\begin{array}{l}\text { Pohlia proligera (Kindb.) Broth. } \\
\text { P. camptotrochela var. vestitissima (Sak.) } \\
\text { Ochi }\end{array}$ & & + & + & $1500-4500$ & C & \\
\hline 79 & $\begin{array}{l}\text { Pohlia wahlenbergii (F. Weber \& Mohr.) A.L. } \\
\text { Andrews }\end{array}$ & & & & $1800-2100$ & $\mathrm{R}$ & \\
\hline 80 & $\begin{array}{l}\text { Rhodobryum giganteum (Schwaegr.) Par. } \\
\text { Bryum giganteum (Schwaegr.) Arnott }\end{array}$ & & + & + & $1000-2700$ & $\mathrm{M}$ & $\begin{array}{l}\text { Ornamental and } \\
\text { medicinal uses }\end{array}$ \\
\hline 81 & $\begin{array}{l}\text { Rhodobryum laxe-limbatum (Ochi) Z. Iwats. } \\
\text { \& Takaki } \\
\text { Bryum laxe-limbatum Hampe ex. Ochi }\end{array}$ & & & + & $2500-2600$ & $\mathrm{R}$ & \\
\hline 82 & $\begin{array}{l}\text { Rhodobryum roseum (Hedw.) Limpr. } \\
\text { Bryum roseum Hedw. }\end{array}$ & & + & & $1000-4000$ & $\mathrm{M}$ & Ornamental uses \\
\hline
\end{tabular}




\section{Discussion}

A total of 82 species including three subspecies and five varieties belonging to seven genera of Bryaceae have been reported in this study mainly from the lowland to the alpine zones of Nepal. The species number of each genus is presented below in bar diagram (Fig.1) and percentage in pie chart (Fig. 2). Pohlia microstoma (Harv.) Ochi has been recorded from 2800-6250 m. This was reported for the first time by Norkett (6910) in 1961 and is deposited at the British Museum, London. The lowland members of Bryaceae which occur below 1000 $\mathrm{m}$ and distributed up to $5100 \mathrm{~m}$ are Brachymenium acuminatum Harv. (250-1800 m), Bryum apiculatum Schwaegr (150-4400 m), B. argenteum Hedw, (800-5100 m), B. caespiticum Hedw, (300-4800 m), B. capillare Hedw. (900-1300 m), B. coronatum Schwaegr. (150-1100 m), B. clavatum (350-4850 m), B. dichotomus Hedw. (500-4600 m), Pohlia leucostomoides (Broth.) Ochi (550 $3400 \mathrm{~m}$ ) and three species of Rhodobryum. Bryum coronatum is the most common species of Bryaceae which occurs in tropical regions only. This study also came up with a list of ten Type Specimens. Studies have shown that the high diversity of Bryaceae was found at subtropical to temperate zones than tropical and alpine regions. Some members of this family carry significant economical values. Such species include Rhodobryum giganteum (Giant Bryum) is used to cure angina in China (Glime and Keen, 1984). Similarly, Bryum algovicum Sendtn. ex C. Muell. (Drooping Thread Moss) growing in sandy area has an amazing ability to check erosion (Crum, 1973). The experiment conducted in Japan testified that Bryum argenteum can be used as a bioindicator of the atmospheric pollution (Taoda 1972). Glime and Saxena (1991) have mentioned that certain species of Bryum can alleviate pain caused mainly by cut and burn.

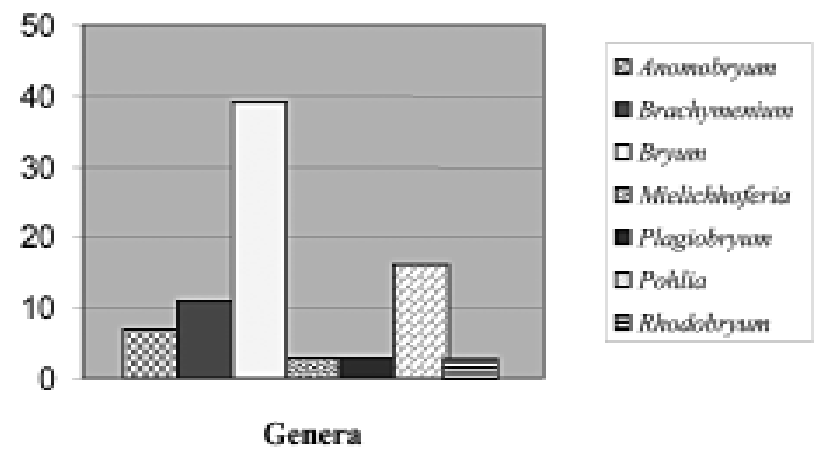

Fig.1. Bar diagram showing species diversity of Bryaceae 

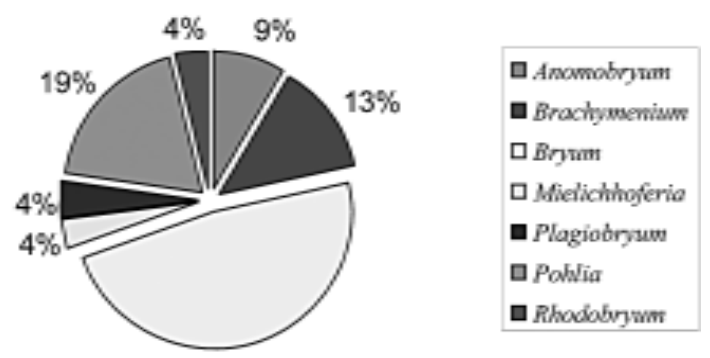

$47 \%$

Fig. 2. Pie chart showing percentage of species of Bryaceae

Anomobryum - 7 species

Brachymenium -11 species

Bryum -39 species

Mielichhoferia - 3 species

Plagiobryum -3 species

Pohlia -16 species

Rhodobryum -3 species

\section{Acknowledgement}

Our thanks go to Prof. Dr. P. K. Jha, Head, Central Department of Botany, Tribhuvan University for providing us laboratory facilities and Prof. K. B. Shah, Chief of the Natural History Museum for his cooperation in our research.

\section{References}

Allen, B. (2002). Moss flora of Central America Part 2. Encalyptaceae-Orthotrichaceae. Missouri Botanical Garden Press: 289-420.

Andrew, A. L. (1935). Bryaceae. In: A. J. Grout (ed.), Moss Flora of North America North of Mexico 2(3): 184-210. Published by the author, Newfane, Vermont.

Brummit, R. K. and Powell, C.E. (1992). Authors of Plant Names. Royal Botanic Garden, Kew. Chopra, R.S. (1975). Taxonomy of Indian Mosses. CSIR, New Delhi, India.

Crum, H. (1973). Mosses of the Great lakes forest. Contribution from the Michigan Herbarium 10: 404.

Eddy, A. (1996). A Handbook of Malaysian Mosses. The Nat. Hist. Mus., London 3: 117-183. Gangulee, H.C. (1974). Mosses of Eastern India and Adjacent Regions 4: 888-1022. Calcutta. 
Glime, J. M. and Keen, R.E. (1984). The importance of Bryophytes in a Man-centered world. J. Hatt. Bot. Lab. 55:133-146.

Glime, J. M. and Saxena, D. (1991). Uses of Bryophytes. Today Tomorrow's Pubs., India Hedderson, T.A. \& Harold, A.S. (1990). Plagiobryum duthiei, a new species from Nepal. Linbergia 16:51-54.

Higuchi, H. and Takaki, N. (1990). Mosses from Nepal collected by Botanical Expeditions of National Science Museum, Tokyo. Cryptogams of the Himalaya Central and Eastern

Nepal M. Watanabe and S. B. Malla (eds.). National Sci. Mus., Tsukuba, Japan 2:132-139.

Iwatsuki, Z. (1979a). Mosses from Central Nepal collected by the Kochi Himalaya Expedition, 1976. Journ. Hatt. Bot. Lab. 46:376-377.

Iwatsuki, Z. (1979b). Mosses from Eastern Nepal collected by Himalayan expedition of Chiba University 1977. Journ. Hattori Bot. Lab. 46:295-297.

Karczmarz, K. (1981). Bryophytes from Nepal. Lindbergia 2:126-130.

Kattel, L.P. and Adhikari, M.K. (1992). Mosses of Nepal (List and References). Nat. Hist. Soc. of Nepal:11-18

Long, D. G. (1995). The Musci Indici: Its Authors, Types and Localities. Bot. J. Linnean Soc. 119:7-16.

Mitten, W. (1858). Musci Indiae Orientalis, an Enumeration of the Mosses of the East Indies. Proc. Journ. Linn. Soc. of London: 65-75.

Noguchi, A. (1966). Musci. In Hara, H. (Eds.). The Flora of Eastern Himalaya. Univ. Mus., Univ. of Tokyo Vol.1:555-559.

Noguchi, A., Takaki, N. and Inoue, H. (1966). Bryophytes collected by Dr. K. Yoda in Eastern Nepal. Bull. Nat. Sci. Mus. 9(3):367-368.

Noguchi, A. (1971). Musci. In Hara, H. (Eds.). The Flora of Eastern Himalaya. Univ. Mus., Univ. of Tokyo Vol. 2:244-245.

Nyholm, E. (1974). Illustrated Moss Flora of Fennoscandia. The Bot. Soc. of London 4: 189-259.

Pradhan, N., (2000a). Materials for a Checklist of Bryophytes of Nepal. The Nat. Hist. Mus., London: 27-33.

Pradhan, N. (2000b). Bryophytes of Phulchowki, Central Nepal. Journ. Nat. Hist. Mus. 19: 64-65.

Pradhan, N and Shrestha, K. 2002. Alpine Bryoflora of Nepal. Proc. Int. Seminar on MountainKathmandu:545-462.

Taoda, H. (1972). Mapping of atmospheric pollution in Tokyo based upon epiphytic bryophytes. Jap. Journ. Ecol. 22:125-133.

Watson, E.V. and Richards, P. (1968). British Mosses and Liverworts. Cambridge Univ. Press.

Website МОВОт: http://www.mobot.org/МОВОт/Moss/China/china-b.shtml 


\section{Abbreviations:}

BM British Museum

C Central, Common

E East, Edinburgh Herbarium

GL Glasgow University Herbarium

W West

T Type

M Medium

m Meter

R Rare

TCD Harvey's Herbarium in Dublin

$+\quad$ Distribution

\section{Author's Address}

${ }^{1}$ Mrs. Nirmala Pradhan and ${ }^{2}$ Dr. Sanu Devi Joshi

${ }^{1}$ Natural History Museum, Tribhuvan University, Swayambhu, Kathmandu, Nepal

${ }^{2}$ Central Department of Botany, Tribhuvan University, Kirtipur, Kathmandu, Nepal 